\title{
Efecto de Dietas Suplementadas con Fitasa y 25-Hidroxico- lecalciferol sobre el Comportamiento Productivo, Mineralización e Integridad de las Tibias en Pollos de Carne
}

\author{
EfFect of Diets Supplemented with Phytase and 25-Hydroxycholecalciferol \\ on Productive Performance, and Mineralization and Integrity of \\ Tibias IN BROILERS
}

Gabriela Peceros P. ${ }^{1}$, Otto Zea M. ${ }^{1}$, Carlos Vílchez P. ${ }^{1,2}$

\section{RESUMEN}

El estudio tuvo por objetivo evaluar la suplementación de la enzima fitasa y la vitamina 25-hidroxicolecalciferol $\left(25-\mathrm{OHD}_{3}\right)$ en dietas para pollos de carne a través de su respuesta productiva y características de la tibia. Se utilizaron 200 pollos machos de la Línea Cobb 500, distribuidos en cuatro tratamientos con cinco repeticiones y 10 animales por repetición: T1, control; T2, iso-nutritivo como T1, considerando la matriz de la fitasa ( $0.178 \%$ Ca y $0.15 \% \mathrm{Pd}$ - fósforo disponible); $\mathrm{T} 3$, iso-nutritivo como $\mathrm{T} 1$, considerando la matriz de la $25-\mathrm{OHD}_{3}(0.1 \%$ Ca y $0.05 \% \mathrm{Pd})$ y T4, iso-nutritivo como $\mathrm{T} 1$, considerando las matrices de la fitasa y $25-\mathrm{OHD}_{3}$. El peso de los pollos y el consumo de alimento fueron registrados los días 7, 14 y 21 . El día 21 se midió la longitud y peso de la tibia de 12 pollos por tratamiento. Las tibias izquierdas fueron procesadas para determinar el contenido de ceniza, calcio y fósforo. No se encontró diferencia estadística en peso corporal, ganancia de peso diario o rendimiento de carcasa entre tratamientos. T3 resultó con valores menores en el largo, peso y densidad de la tibia $(\mathrm{p}<0.05)$ en comparación con los demás tratamientos. Los porcentajes de cenizas y calcio de la tibia de pollos en T2 y T4 fueron menores que los pollos en T1 o T3 $(\mathrm{p}<0.05)$. El porcentaje de fósforo de la tibia de pollos en T2, T3 y T4 fue similar al grupo control (T1). Se concluye que la enzima fitasa, la vitamina $25-\mathrm{OHD}_{3}$ y la combinación de ambas como suplemento en las dietas de pollos de carne no tuvieron un efecto positivo en el comportamiento productivo de las aves, ni contribuyeron a una eficaz mineralización de la tibia.

Palabras clave: 25 -hidroxicolecalciferol, fitasa, pollos, ceniza, calcio, tibia

\footnotetext{
${ }^{1}$ Departamento Académico de Nutrición, Facultad de Zootecnia, Universidad Nacional Agraria La Molina, Lima, Perú

${ }^{2}$ E-mail: cvilchezp@lamolina.edu.pe
}

Recibido: 30 de enero de 2016

Aceptado para publicación: 16 de mayo de 2016 
The study aimed to evaluate the effects of the inclusion, alone or in combination, of phytase and 25-hydroxycholecalciferol vitamin $\left(25-\mathrm{OHD}_{3}\right)$ on the productive performance and tibia characteristics of male broilers from 1 to 21 day of age. A total of 200 male chicks of the Cob 500 Line were distributed in four treatments with five replicates and 10 birds per repetition. The treatments were: $\mathrm{T} 1$, Control; $\mathrm{T} 2$, Isonutritive as $\mathrm{T} 1$ considering phytase matrix $(0.178 \% \mathrm{Ca}$ and $0.15 \% \mathrm{AP}$ - available phosphorous); $\mathrm{T} 3$, Isonutritive as $\mathrm{T} 1$ considering $25-\mathrm{OHD}_{3}$ matrix $(0.1 \% \mathrm{Ca}$ and $0.05 \% \mathrm{AP})$ and $\mathrm{T} 4$, Isonutritive as $\mathrm{T} 1$ considering both phytase and $25-\mathrm{OHD}_{3}$ matrixes. Broilers were weighed and feed intake recorded on days 7 , 14 , and 21 . On day 21 , lengths and weights of tibias of twelve chicks per treatment were measured. Left tibias were tested for ash, calcium and phosphorus contents. No statistical difference was found in body weight, daily weight gain or carcass yield between treatments. T3 showed lower bone density, shorter and lighter tibias than those of other treatments $(\mathrm{p}<0.05)$. Ash contents and calcium concentrations in tibias from birds fed T2 or T4 were lower than those on T1 or T3 $(\mathrm{p}<0.05)$. Phosphorus concentration in tibia from broilers fed $\mathrm{T} 2$, T3, and T4 were similar to those fed T1. In conclusion, the phytase enzyme, vitamin 25$\mathrm{OHD}_{3}$ and the combination of both as a supplement in diets of broilers neither had positive effect on the productive performance of broilers nor contributed to effective mineralization of the tibia.

Key words: 25-hydroxycholecalciferol, phytase, broilers, ash, calcium, tibia

\section{INTRODUCCIÓN}

Los avances en selección genética en la industria avícola han mejorado la tasa de crecimiento en los pollos de carne. Sin embargo, se siguen presentando limitaciones con respecto al aprovechamiento de ciertos nutrientes como el fósforo en los ingredientes de origen vegetal. El ácido fítico es considerado como un agente antinutricional, puesto que las aves no disponen de la enzima correspondiente para hidrolizarla y liberar los elementos ligados, principalmente fósforo. Entre el 50-80\% de este elemento se encuentra ligado a las moléculas de ácido fítico (Kocabagli, 2001; Godoy y Chicco, 2005; Liem et al., 2009). Además, este ácido puede formar complejos con otros minerales, principalmente el calcio (Shirley y Edwards, 2003; Selle et al., 2009; Khalid et al., 2013). En la actualidad se vienen usando diferentes fuentes de fitasas comerciales con el propósito de aprovechar los nutrientes ligados en el ácido fitico (Khalid et al., 2013).

Por otro lado, la vitamina D cumple un rol importante en el metabolismo de calcio y fósforo, particularmente en el desarrollo y mantenimiento del esqueleto, así como también sobre la calidad de la cáscara de huevo (Cunningham, 2009). El metabolito de la vitamina D, 25-hidroxi vitamina $\mathrm{D}_{3}\left(25-\mathrm{OHD}_{3}\right)$, es tan eficiente como la vitamina $\mathrm{D}_{3}$ misma, de allí que se emplean con frecuencia en programas de alimentación de los pollos de carne y de otras especies de aves comerciales (McDowell, 2000; Cunningham, 2009), por su influencia en el metabolismo del calcio (Edwards, 2002; Coto et al., 2008). Dado que tanto la vitamina $\mathrm{D}$ como las fitasas influyen en la mineralización de los huesos, es necesario evaluar su uso mediante indicadores sensibles. En este caso, la tibia es el hueso de más rápido crecimiento en el cuerpo y es considerado como el más sensible a las deficiencias de calcio y fósforo (Yan et al., 2005). 
Cuadro 1. Composición porcentual y valor nutricional de las dietas experimentales empleadas en pollos de carne en la etapa de 1 a 21 días de edad

\begin{tabular}{lcccc}
\hline & \multicolumn{4}{c}{ Tratamientos $^{1}$} \\
\cline { 2 - 5 } Ingredientes & $\mathrm{T} 1$ & $\mathrm{~T} 2$ & $\mathrm{~T} 3$ & $\mathrm{~T} 4$ \\
\hline Maíz amarillo & 59.825 & 61.276 & 60.489 & 61.940 \\
Torta de soya & 32.223 & 31.967 & 32.106 & 31.849 \\
Aceite crudo de soya & 4.277 & 3.801 & 4.059 & 3.583 \\
Carbonato de calcio & 0.810 & 0.870 & 0.731 & 0.791 \\
Fo sfato dicálcico & 1.578 & 0.786 & 1.306 & 0.515 \\
Sal común & 0.466 & 0.465 & 0.465 & 0.465 \\
Metionina DL & 0.258 & 0.256 & 0.257 & 0.255 \\
Lisina HCL & 0.144 & 0.149 & 0.146 & 0.151 \\
Treonina L & 0.050 & 0.051 & 0.051 & 0.051 \\
Premezcla vitamínico-mineral ${ }^{2}$ & 0.120 & 0.120 & 0.120 & 0.120 \\
Cloruro de colina 60 & 0.100 & 0.100 & 0.100 & 0.100 \\
Antox plus (antioxidante) & 0.050 & 0.050 & 0.050 & 0.050 \\
Zinc Bacitracina 10 (antibiótico) & 0.050 & 0.050 & 0.050 & 0.050 \\
Mycosorb (secuestrante) & 0.050 & 0.050 & 0.050 & 0.050 \\
Vitamina 25-OHD 3 & - & - & 0.020 & 0.020 \\
Enzima fitasa & - & 0.010 & - & 0.010 \\
\hline Nutrientes (calculado) & & & & \\
\hline E. Metabolizable, (kcal/kg) & 3072 & 3072 & 3072 & 3072 \\
Proteína total, \% & 20.5 & 20.5 & 20.5 & 20.5 \\
Lisina digestible, \% & 1.12 & 1.12 & 1.12 & 1.12 \\
Metionina digestible, \% & 0.55 & 0.55 & 0.55 & 0.55 \\
Metionina + cistina digestible, \% & 0.84 & 0.84 & 0.84 & 0.84 \\
Triptófano digestible, \% & 0.21 & 0.21 & 0.21 & 0.21 \\
Treonina digestible, \% & 0.73 & 0.73 & 0.73 & 0.73 \\
Calcio, \% & 0.87 & 0.87 & 0.87 & 0.87 \\
Fósforo disponible, \% & 0.44 & 0.44 & 0.44 & 0.44 \\
Sodio, \% & 0.20 & 0.20 & 0.20 & 0.20 \\
\hline
\end{tabular}

${ }^{1} \mathrm{~T} 1$ : dieta basal (control); T2: T1 + la matriz de fitasa (0.178\% Ca y $0.15 \%$ Pd); T3: T1 + la matriz de $25-\mathrm{OHD}_{3}(0.1 \%$ Ca y $0.05 \% \mathrm{Pd})$; T4: T1 + ambas matrices de fitasa y $25-\mathrm{OHD}_{3}$

Las formas de participación en la dietas pueden variar, sea como un ingrediente con o sin matrices (Tamim et al., 2004). En algunos casos solo puede ser considerada la fitasa o la vitamina 25-hydroxicolecalciferol $\left(25-\mathrm{OHD}_{3}\right)$ y en otros casos se emplean ambos productos. Este hecho no facilita una interpretación adecuada de los resultados de las investigaciones, de modo que se requiere establecer la forma en que se usan estos productos en la formulación de las dietas. 
El objetivo del presente estudio fue determinar el efecto de la inclusión de una fitasa y de $25-\mathrm{OHD}_{3}$ comerciales, considerando sus respectivas matrices nutricionales, sobre la respuesta productiva y mineralización e integridad de las tibias de pollos de carne.

\section{Materiales y Métodos}

El presente estudio se llevó a cabo en el Laboratorio de Investigación en Nutrición y Alimentación de Aves (LINAA) de la Universidad Nacional Agraria de La Molina (UNALM), Lima, Perú. Se emplearon 200 pollos BB de la Línea Cobb 500 machos de un día de edad. Las aves fueron distribuidas al azar en 4 tratamientos de 50 pollos cada uno. Cada tratamiento constó de 5 repeticiones con 10 pollos por repetición. El tiempo de crianza fue de 1 a 21 días de edad. Las aves estuvieron alojadas en dos jaulas metálicas de malla galvanizada (baterías) de cinco pisos y con dos divisiones por piso, y con comederos y bebederos lineales tipo canaleta en la parte externa.

Los tratamientos fueron: $\mathrm{T} 1$, dieta basal (control); T2, T1 + la matriz de fitasa $(0.178 \%$ Ca y $0.15 \% \mathrm{Pd}$ - fósforo disponible); T3, T1 + la matriz de $25-\mathrm{OHD}_{3}(0.1 \%$ Ca y $0.05 \%$ Pd); T4, T1 + ambas matrices de fitasa y 25 $\mathrm{OHD}_{3}$. La enzima fitasa fue utilizada en una dosis de $100 \mathrm{mg}$ del suplemento comercial $/ \mathrm{kg}$ de alimento (1000 unidades de fitasa Phyzyme por kilogramo [FTU/kg]), mientras que la dosis de la vitamina $25-\mathrm{OHD}_{3}$ fue de $200 \mathrm{mg}$ del suplemento comercial $/ \mathrm{kg}$ de alimento $\left(69 \mu \mathrm{g}\right.$ de $\left.25-\mathrm{OHD}_{3} / \mathrm{kg}\right)$. Las dietas experimentales fueron formuladas siguiendo las especificaciones nutricionales de la Línea Cobb 500 (Cobb-Vantress, 2002), usando el programa comercial DAPP N-utrition v. 2.0 (2003). La composición y valor nutricional calculado de la dieta basal se muestran en el Cuadro 1.
Al finalizar el periodo experimental se sacrificaron 12 pollos por cada tratamiento para la extracción de ambas tibias por ave. Para esto, las tibias fueron retiradas, identificadas e introducidas en malla mosquitera en forma individual. Dicho envoltorio fue sometido a agua hirviendo por $15 \mathrm{~min}$ para remover el tejido del hueso (Applegate y Lilburn, 2002), procedimiento que no altera el contenido mineral ni la densidad del hueso y permite retirar el $80 \%$ de la grasa (Almeida y Bruno, 2006). Los tejidos y cartílagos presentes en las zonas de articulación fueron retirados siguiendo el procedimiento de Baumel y Witmer (1993). Los huesos fueron limpiados con papel toalla y colocados en un recipiente. Una semana después se hicieron las mediciones respectivas.

Se determinó el peso vivo, ganancia de peso vivo a los 7, 14 y 21 días de edad y el consumo voluntario de alimento para lo que se utilizó una balanza digital de plataforma con precisión de $0.01 \mathrm{~g}$. La conversión alimenticia se determinó a los 21 días de edad, dividiendo el consumo del alimento entre la ganancia de peso.

Se tomaron las características morfométricas de la tibia derecha (diámetro de la diáfisis, largo, volumen y peso) e indicadores de mineralización de la tibia como densidad ósea (Rath et al., 2000), índice de Seedor (Seedor et al., 1991), índice de Quetelet (Rutten et al., 2002; Martínez, 2012), índice de robusticidad (Kokabagli, 2001; Mutus et al., 2006; Martínez, 2012) y porcentajes de cenizas, calcio y fósforo.

La densidad ósea de la tibia se determinó mediante la volumetría del hueso (Quarantelli et al., 2007), considerándose como densidad ósea a la masa de material orgánica e inorgánica en el hueso por unidad de volumen del mismo (Rath et al., 2000). Se calculó dividiendo el peso del hueso (mg) entre el volumen $\left(\mathrm{cm}^{3}\right)$. 
Cuadro 2. Efecto de la enzima fitasa, vitamina 25-hidroxicolecalciferol $\left(25-\mathrm{OHD}_{3}\right)$ o combinación de ambas sobre el comportamiento productivo de pollos de carne en la fase de 1-21 días de edad

\begin{tabular}{lcccc}
\hline Mediciones & T1 & T2 & T3 & T4 \\
\hline Peso vivo inicial (g/ave) & $48.4^{\mathrm{a}}$ & $48.2^{\mathrm{a}}$ & $49.2^{\mathrm{a}}$ & $48.5^{\mathrm{a}}$ \\
Peso vivo final (g/ave) & $972.6^{\mathrm{a}}$ & $978.6^{\mathrm{a}}$ & $975.0^{\mathrm{a}}$ & $986.4^{\mathrm{a}}$ \\
Ganancia de peso en 21 días (g/ave) & $924.2^{\mathrm{a}}$ & $930.5^{\mathrm{a}}$ & $925.8^{\mathrm{a}}$ & $937.9^{\mathrm{a}}$ \\
Consumo de alimento total (g/ave) & $1215.2^{\mathrm{a}}$ & $1206.5^{\mathrm{a}}$ & $1208.6^{\mathrm{a}}$ & $1236.2^{\mathrm{a}}$ \\
Conversión alimenticia acumulada (g/g) & $1.32^{\mathrm{a}}$ & $1.30^{\mathrm{a}}$ & $1.31^{\mathrm{a}}$ & $1.32^{\mathrm{a}}$ \\
\hline
\end{tabular}

${ }^{a, b}$ Superíndices diferentes dentro de filas indican diferencia estadística $(p<0.05)$.

${ }^{1}$ Tratamientos: T1: control; $\mathrm{T} 2$ : dieta conteniendo fitasa; $\mathrm{T3}$ : dieta conteniendo $25-\mathrm{OHD}_{3}$; T4: dieta conteniendo fitasa $+25-\mathrm{OHD}_{3}$

Cuadro 3. Efecto de la suplementación con fitasa y $25-\mathrm{OHD}_{3}$ en dietas para pollos de carne (1-21 días) sobre los indicadores de la mineralización ósea de la tibia

\begin{tabular}{lcccc}
\hline Mediciones & $\mathrm{T}^{1}$ & $\mathrm{~T} 2$ & $\mathrm{~T} 3$ & $\mathrm{~T} 4$ \\
\hline Densidad del hueso $(\mathrm{g} / \mathrm{ml})$ & $1.03^{\mathrm{a}}$ & $1.07^{\mathrm{a}}$ & $0.91^{\mathrm{b}}$ & $1.05^{\mathrm{a}}$ \\
Índice modificado de Seedor $(\mathrm{mg} / \mathrm{mm})$ & $37.640^{\mathrm{a}}$ & $34.859^{\mathrm{b}}$ & $34.115^{\mathrm{b}}$ & $35.925^{\mathrm{ab}}$ \\
Índice de Quetelet $\left[\mathrm{mg} /(\mathrm{mm})^{2}\right]$ & $5.610^{\mathrm{a}}$ & $5.151^{\mathrm{b}}$ & $5.171^{\mathrm{b}}$ & $5.292^{\mathrm{b}}$ \\
Índice de robusticidad $\left[\mathrm{cm} /(\mathrm{g})^{?}\right]$ & $4.934^{\mathrm{b}}$ & $5.088^{\mathrm{a}}$ & $5.037^{\mathrm{a}}$ & $5.044^{\mathrm{a}}$ \\
Análisis químico & & & & \\
$\quad$ Ceniza (\%) & $44.66^{\mathrm{a}}$ & $42.90^{\mathrm{b}}$ & $43.42^{\mathrm{ab}}$ & $42.40^{\mathrm{b}}$ \\
Calcio (\%) & $37.16^{\mathrm{ab}}$ & $36.40^{\mathrm{b}}$ & $37.76^{\mathrm{a}}$ & $36.85^{\mathrm{ab}}$ \\
Fósforo (\%) & $17.41^{\mathrm{a}}$ & $17.29^{\mathrm{a}}$ & $17.29^{\mathrm{a}}$ & $17.60^{\mathrm{a}}$ \\
\hline
\end{tabular}

${ }^{a, b}$ Superíndices diferentes dentro de filas indican diferencia estadística $(p<0.05)$

${ }^{1}$ Tratamientos: T1: control positivo; T2: dieta conteniendo fitasa; T3: dieta conteniendo 25- OHD3; T4: dieta conteniendo fitasa + 25-OHD3

El índice de Seedor se determinó dividiendo el peso del hueso (mg) entre su largo $(\mathrm{mm})$. El Índice de masa corporal o de Quetelet se obtuvo dividiendo el peso del hueso (mg) entre la longitud al cuadrado del mismo $\left(\mathrm{mm}^{2}\right)$. El índice de robusticidad se obtuvo dividiendo el largo del hueso $(\mathrm{mm})$ entre el peso $(\mathrm{g})$ elevado a la potencia $1 / 3$.
Los porcentajes de ceniza se obtuvieron por calcinación de las tibias izquierdas. Para esto, fueron colocadas en un horno $\left(55^{\circ} \mathrm{C}\right)$ por $72 \mathrm{~h}$ para secarlas, luego fueron molidas en un molino de bolas y colocadas en un horno de mufla a $550^{\circ} \mathrm{C}$ por $3 \mathrm{~h}$. La ceniza fue cuantificada para calcio y fósforo por la técnica de Silva y Queiroz (2002). 
Se empleó un diseño completamente al azar con cuatro tratamientos, cinco repeticiones por tratamiento y 10 aves por repetición. El análisis de varianza se llevó a cabo usando el programa Statistical Analysis System (SAS, 1999) y la comparación de medias se realizó utilizando la Prueba de Duncan.

\section{Resultados y Discusión}

El Cuadro 2 muestra el efecto de los tratamientos sobre el comportamiento productivo de los pollos de carne. No se observaron diferencias significativas entre tratamientos en el peso vivo final y en la ganancia de peso a los 21 días de edad. Estos resultados guardan relación con otros estudios (Driver et al., 2005a; Selle y Ravindran, 2006; Santos et al., 2008; Zhou et al., 2008; Liem et al., 2009), quienes tampoco encontraron diferencias estadísticas en el peso vivo a los 21 días de edad en pollos suplementados con fitasas y $25-\mathrm{OHD}_{3}$ (T4).

Los resultados sobre consumo de alimento y conversión alimenticia tampoco mostraron diferencias estadísticas entre tratamientos (Cuadro 2), lo cual indica que estos valores fueron similares entre el grupo control (T1) y aquellos suplementados, sea con fitasa (T2), 25-OHD 3 (T3) o con ambos (T4). Similares resultados fueron observados por Hermes et al. (2013) en pollos alimentados con dietas conteniendo fitasa (1000 FYT/kg [Unidad de fitasa Ronozyme]) más 25-OHD $(69 \mu \mathrm{g} / \mathrm{kg}$ ), y por otros investigadores (Ahmad et al., 2000; Driver et al., 2005b; Roberson et al., 2005; Santos et al., 2008).

Las características morfométricas de la tibia por tratamiento se encuentran en el Cuadro 3. Se observa una disminución significativa de la densidad del hueso en T3 en relación con los otros tratamientos $(\mathrm{p}<0.05)$, donde no hubo variación entre ellos. La densidad ósea es un indicador de la fortaleza del hueso y refleja el contenido mineral del hueso (Rath et al., 2000), por lo cual, a menor densidad ósea aumenta el riesgo de fracturas. Estos resultados indican una menor fortaleza de la tibia en los pollos suplementados con 25$\mathrm{OHD}_{3}$. Rutherfurd et al. (2012), trabajando con suplementación con fitasa en dietas para pollos de carne con bajo nivel de fósforo disponible $(0.35 \%)$ y nivel normal de calcio, obtuvieron similar 'densidad mineral' de la tibia $\left(\mathrm{mg} / \mathrm{cm}^{2}\right)$ en comparación con pollos que recibieron una dieta con niveles normales de calcio y fósforo.

En este estudio, los tratamientos con suplementación (T2, T3, T4) obtuvieron valores más bajos en los índices de Seedor y de Quetelet y valores altos en el índice de robusticidad con respecto al T1 (control), sugiriendo que las dietas experimentales no incrementaron el grado de mineralización y desarrollo del hueso. No obstante, el estudio de Kocabagli (2001), suplementando varios niveles de fitasa $(300,500$ y $700 \mathrm{U}$ de fitasa $/ \mathrm{kg}$ ) en dietas para pollos de carne a base de maízsoya, obtuvo significativos incrementos en el índice de Seedor y bajos valores en el índice de robusticidad. Asimismo, en el estudio de García et al. (2013) con dietas a base de maíz-soya para pollos de carne, el índice de Seedor de la tibia y otros parámetros óseos fueron similares entre el grupo alimentado con la dieta conteniendo vitamina $\mathrm{D}_{3}$ (dieta control) y los otros grupos alimentados con dietas conteniendo $25-\mathrm{OHD}_{3}, 1,25-(\mathrm{OH})_{2} \mathrm{D}_{3} \mathrm{O}$ $1 \alpha-\mathrm{OHD}_{3}$.

Los porcentajes de ceniza, calcio y fósforo de la tibia izquierda de los pollos de cada tratamiento se encuentran en el Cuadro 3. La suplementación de la fitasa (T2) y la combinación de fitasa con $25-\mathrm{OHD}_{3}$ (T4) disminuyeron significativamente $(\mathrm{p}<0.05)$ el valor de cenizas de la tibia en relación con la dieta control (T1). Sin embargo, la suplementación con $25-\mathrm{OHD}_{3}$ (T3) tuvo un efecto similar a la dieta control. Además, la dieta T3 tuvo un valor de cenizas similar a las dietas T2 y T4. Los valores de cenizas se encuentran en el rango $42.4-44.7 \%$. Por otro lado, se encon- 
tró que la suplementación con fitasa (T2) redujo significativamente $(\mathrm{p}<0.05)$ el valor del calcio en comparación con la suplementación con $25-\mathrm{OHD}_{3}$ (T3) $(36.4 \%$ versus $37.8 \%)$. Los valores de calcio en la dieta suplementada con fitasa y $25-\mathrm{OHD}_{3}$ (T4) y en la dieta control (T1) fueron similares.

Los resultados del presente estudio coinciden con Zhou et al. (2008) en pollos de carne (0-28 días), quienes obtuvieron bajo porcentaje de calcio de la tibia en el grupo suplementado con fitasa, aunque el porcentaje de cenizas de la tibia fue similar al del grupo control, concluyendo que el nivel de calcio $(0.67 \% \mathrm{Ca})$ contenido en las dietas suplementadas con fitasa fue inferior a la cantidad necesaria para cubrir el requerimiento de calcio en los pollos. Asimismo, Hermes et al. (2013), trabajando con dietas suplementadas con fitasa $(1000 \mathrm{FYT} / \mathrm{kg})$ y $25-\mathrm{OHD}_{3}$ $(69 \mu \mathrm{g} / \mathrm{kg})$, pero deficientes en calcio $(0.72 \%)$ $\mathrm{y}$ fósforo disponible $(0.30 \%)$, obtuvieron bajo porcentaje de cenizas de la tibia en comparación con pollos con la dieta control.

Al parecer, la fitasa exógena no reduce el requerimiento de calcio o libera una cantidad mínima de calcio a partir de las dietas para pollos de carne (Mitchell y Edwards, 1996; Yan et al., 2006). Ahmad et al. (2000) observaron que pollos (0-28 días) alimentados con una dieta conteniendo fitasa y con nivel normal de calcio $(0.1 \% \mathrm{Ca})$ y bajo de fósforo no fítico $(0.36 \%)$ obtuvieron similar contenido de ceniza, calcio y fósforo del hueso en relación con pollos alimentados con una dieta conteniendo niveles normales de calcio $\mathrm{y}$ fósforo.

En el presente estudio, la respuesta productiva de las dietas conteniendo fitasa (T2) y la combinación de fitasa más $25-\mathrm{OHD}_{3}(\mathrm{~T} 4)$ fueron similares al grupo control, y los porcentajes de cenizas y calcio de la tibia mostraron niveles menores con respecto al tratamiento control (T1). Esto pudo ser debido a que los niveles de calcio y fósforo requeridos en la dieta para maximizar la respuesta pro- ductiva de los pollos de carne no son suficientes para obtener un adecuada mineralización de los huesos (McDowell, 2003). Por otro lado, Bar et al. (2003) indican que el nivel adecuado de calcio para la mineralización de los huesos es mayor que lo requerido para maximizar la ganancia de peso vivo, pero que los niveles de fósforo requeridos para la obtención de una alta ganancia de peso son adecuados para una buena mineralización del hueso.

En el presente trabajo, el porcentaje de fósforo de las tibias fue similar entre los cuatros tratamientos, lo cual coincide con otros autores, que sugieren que la suplementación con fitasa o con $25-\mathrm{OHD}_{3}$ incrementan la absorción del fósforo a nivel intestinal y no afectan negativamente el porcentaje de fósforo de los huesos de pollos de carne (Applegate et al., 2003; Fritts y Waldroup, 2005; Driver et al., 2005a; Santos et al., 2008).

\section{Conclusiones}

La enzima fitasa, la vitamina $25-\mathrm{OHD}_{3}$ y la combinación de ambas como suplemento en las dietas de pollos de carne no tuvieron un efecto positivo en el comportamiento productivo de las aves, ni contribuyeron a una eficaz mineralización de la tibia.

\section{Literatura Citada}

1. Ahmad T, Rassol S, Sarwar M, Haq A, Hassan, Z. 2000. Effect of microbial phytase produced from a fungus Aspergillus niger on bioavailability of phosphorus and calcium in broiler chickens. Anim Feed Sci Technol 83: 103-114. doi: 10.1016/S0377-8401(99) 00122-4

2. Almeida P, Bruno LDG 2006. Bone mineral density: review. Braz J Poultry Sci 8: 69-73 
3. Applegate TJ, Angel R, Classen HL. 2003. Effect of dietary calcium, 25hydroxycholecalciferol, or bird strain on small intestinal phytase activity in broiler chickens. Poultry Sci 82: 1140-1148. doi: $10.1093 / \mathrm{ps} / 82.7 .1140$

4. Applegate TJ, Lilburn MS. 2002. Growth of the femur and tibia of a commercial broiler line. Poultry Sci. 81: 1289-1294. doi: 10.1093/ps/81.9.1289

5. Bar A, Shinder D, Yosefi S, Vax E, Plavnik I. 2003. Metabolism and requirements for calcium and phosphorus in the fast-growing chicken as affected by age. Brit J Nutr 89: 51-60. doi: 10.1079/BJN2002757

6. Baumel JJ, Witmer LM. 1993. Osteología. En: Baumel JJ, King AS, Breazile GE, et al. (eds). Handbook of avian anatomy: nomina anatomica avium. $2^{\text {nd }}$ ed. Cambridge Massachusetts: Nuttall Ornithological Club. p 45-132.

7. Cobb-Vantress. 2002. Cobb 500 Breeder management guide. Cobb Vantress Inc. [Internet]. Available in: http://www.cobb-vantress.com/docs/ default-source/cobb-500-guides/ Cobb500_Broiler_Performance And_Nutrition_Supplement.pdf

8. Coto C, Yan F, Cerrate $S$, Wang $Z$, Sacakli P, Halley JT, Wiernusz, CJ, et al. 2008. Effects of dietary levels of calcium and nonphytate phosphorus in broiler starter diets on live performance, bone development and growth plate conditions in male chicks fed a cornbased diet. Int J Poultry Sci 7: 638-645. doi: 10.3923/ijps.2008.638.645

9. Cunningham GJ, Klein GB. 2009. Fisiología veterinaria. $4^{\circ}$ ed. España: Elsevier. $720 \mathrm{p}$.

10. DAPP N-utrition. 2003. Software para formulación de raciones a mínimo costo. Versión 2.0. Argentina. [Internet]. Disponible en: http://www.dapp.com.ar/ nut home.htm

11. Driver JP, Pesti GM, Bakalli RI, Edwards HM. 2005a. Phytase and $1 \alpha-$ hydroxycholecalciferol supplementation of broiler chickens during the starting and growing/finishing phases. Poultry Sci 84: 1616-1628. doi: 10.1093/ps/84.10.1616

12. Driver JP, Pesti GM, Bakalli RI, Edwards HM. 2005b. Effects of calcium and nonphytate phosphorus concentrations on phytase eficacy in broiler chicks. Poultry Sci 84: 1406-1417. doi: $10.1093 / \mathrm{ps} / 84.9 .1406$

13. Edwards HM Jr. 2002. Studies on the efficacy of cholecalciferol and derivatives for stimulating phytate utilization in broilers. Poultry Sci 81: 1026-1031.

14. Fritts CA, Waldroup PW. 2005. Comparison of cholecalciferol and 25hydroxycholecalciferol in broiler diets designed to minimize phosphorus excretion. J Appl Poultry Res 14: 156166. doi: 10.1093/japr/14.1.156

15. García AF, Murakami A, Duarte C, Ospina I, Picoli P, Mangili M. 2013. Use of vitamin $\mathrm{D}_{3}$ and its metabolites in broiler chicken feed on performance, bone parameters and meat quality. AsianAustralas J Anim Sci 26: 408-415. doi: 10.5713/ajas.2012.12455

16. Godoy S, Chicco C. 2005. Utilización del fósforo fítico en la nutrición de los rumiantes. Rev Digital CENIAP Hoy 9. [Internet]. Disponible en: http:// sian.inia.gob.ve/repositorio/revistas_tec/ ceniaphoy/articulos/n9/arti/godoy__/arti/ godoy_s.htm

17. Hermes R, Iglesias BF, Azcona JO, Loli G, Chariere MV, Hernández JM. 2013. Assess the efficacy of a phytase and 25-hydroxycholecalciferol (25OHD3) on mineral metabolism and performance of broilers fed a corn-soybean $\operatorname{diet}$ (Abstract). In: International Poultry Scientific Forum. [Internet]. Available in: http://www.poultryscience.org/psa13/ abstracts/2013-PSA-Abstracts.pdf

18. Khalid MF, Hussain M, Rehman AU, Shazad MA, Sharif M, Rahman ZU. 2013. Broiler performance in response to phytate and supplemented phytase. Iranian J Appl Sci 3: 1-12.

19. Kocabagli N. 2001. The effect of dietary phytase supplementation at different levels on tibial bone 
characteristics and strength in broilers. Turk J Vet Anim Sci 25: 797-802.

20. Liem A, Pesti A, Atencio A, Edwards JR, HM. 2009. Experimental approach to optimize phytate phosphorus utilization by broiler chickens by addition of supplements. Poultry Sci 88 :1655-1665. doi: $10.3382 / p s .2008-00481$

21. Martínez DA. 2012. Evaluación de un producto a base de aceite esencial de orégano sobre la integridad intestinal, la capacidad de absorción de nutrientes y el comportamiento productivo de pollos de carne. Tesis de Magíster. Lima: Univ Nacional Agraria La Molina. 368 p.

22. McDowell LR. 2003. Minerals in animal and human nutrition. $2^{\text {nd }}$ ed. Amsterdam: Elsevier. $644 \mathrm{p}$.

23. Mitchell RD, Edwards HM. 1996. Effects of phytase and 1,25-hydroxycholecalciferol on phytate utilization and the quantitative requirement for calcium and phosphorus in young broiler chickens. Poultry Sci 75: 95-110. doi: 10.3382/ ps.0750095

24. Mutus R, Kocabagli N, Alp M, Acar $N$, Eren M, Gezen SS. 2006. The effect of dietary probiotic supplementation on tibial bone characteristics and strength in broiler. Poultry Sci 85: 16211625. doi: $10.1093 / \mathrm{ps} / 85.9 .1621$

25. Quarantelli A, Cacchioli A, Romanelli S, Righi F, Alpigiani I, Gabbi C. 2007. Effects of different levels of dietary biotin on the performance and bone structure of broiler. Ital J Anim Sci 6: 517. doi: $10.4081 /$ ijas. 2007.5

26. Rath CN, Huff RG, Huff EW, Balog $M J .2000$. Factors regulating bone maturity and strength in poultry. Poultry Sci 79: 1024-1032. doi: 10.1093/ps/ 79.7.1024

27. Roberson KD, Ledwaba MF, Charbeneau RA. 2005. Studies on the efficacy of twenty-five-hydroxycholecalciferol to prevent tibial dyschondroplasia in Ross broilers fed marginal calcium to market age. Int J Poultry Sci 4: 85-90. doi: 10.3923/ijps.2005.85.90
28. Rutten M, Leterrier C, Constantin P, Reiter K, Bessel W. 2002. Bone development and activity in chickens in response to reduced weight-load on legs. Anim Res 51: 327-336. doi: 10.1051/ animres: 2002027

29. Rutherfurd SM, Chung TK, Thomas DV, Zou ML, Moughan PJ. 2012. Effect of a novel phytase on growth performance, apparent metabolizable energy, and the availability of minerals and amino acids in a low-phosphorus corn-soybean meal diet for broilers. Poultry Sci 91: 1118-1127. doi: 10.3382/ ps.2011-01702

30. SAS institute. 1999. SAS user's guide: statistics, v. 8. USA: SAS Institute, Inc. $965 \mathrm{p}$.

31. Santos FR, Hruby M, Pierson EEM, Remus JC, Sakomura NK. 2008. Effect of phytase supplementation in diets on nutrient digestibility and performance in broiler chicks. J Appl Poult Res 17: 191201. doi: 10.3382/japr.2007-00028

32. Seedor JG, Quarruccio HA, Thompson DD. 1991. The bisphosphonate alendronate (MK-217) inhibits bone loss due to ovariectomy in rats. $\mathrm{J}$ Bone Miner Res 6: 339-346. doi: 10.1359/jbmr.2005.20.2.354

33. Selle HP, Cowieson JA, Ravindran V. 2009. Consequences of calcium interactions with phytase and phytase for poultry and pigs. Livestock Sci 124: 126141. doi: 10.1016/j.livsci.2009.01.006

34. Selle HP, Ravindran V. 2006. Microbial phytase in poultry nutrition. Anim Feed Sci Technol 135: 1-41. doi: 10.1016/ j.anifeedsci.2006.06.010

35. Shirley RB, Edwards HM. 2003. Graded levels of phytase past industry standards improves broiler performance. Poultry Sci 82: 671-680. doi: 10.1093/ps/ 82.4 .671

36. Silva DJ, Queiroz AC. 2002. Preparo de solução mineral. In: Silva DJ, Queiroz $\mathrm{AC}$ (eds). Análise de alimentos: métodos químicos e biológicos. Viçosa, Brazil; Ed UFV. p 83-86. 
37. Tamim MN, Angel R, Christman M. 2004. Influence of dietary calcium and phytase on phytate phosphorus hydrolysis in broiler chickens. Poultry Sci 83: 13581367. doi: $10.1093 / \mathrm{ps} / 83.8 .1358$

38. Yan F, Keen CA, Zhang KY, Waldroup $P W$. 2005. Comparison of methods to evaluate bone mineralization. J Appl Poul Res 14: 492-498. doi: 10.1093/japr/ 14.3.492
39. Yan F, Kersey JH, Fritts, CA, Waldroup PW. 2006. Effect of phytase supplementation on the calcium requirement of broiler chicks. Int $J$ Poultry Sci 5: 112-120.

40. Zhou JP, Yang ZB, Yang WR, Wang $X Y$, Jiang SZ, Zhang GG. 2008. Effects of a new recombinant phytase on the performance and mineral utilization of broilers fed phosphorusdeficient diets. J Appl Poult Res 17: 331339. doi: 10.3382/japr.2007-00111 